\title{
Formation and morphological evolution of self-similar 3D nanostructures on weakly interacting substrates
}

\author{
B. Lü, ${ }^{1}$ G. A. Almyras, ${ }^{1}$ V. Gervilla, ${ }^{1}$ J. E. Greene, ${ }^{2,3}$ and K. Sarakinos ${ }^{1, *}$ \\ ${ }^{1}$ Nanoscale Engineering Division, Department of Physics, Chemistry and Biology, Linköping University, SE 581 83, Linköping, Sweden \\ ${ }^{2}$ Thin Film Physics Division, Department of Physics, Chemistry and Biology, Linköping University, SE-581 83 Linköping, Sweden \\ ${ }^{3}$ Materials Science and Physics Departments, University of Illinois, Urbana, Illinois 61801, USA
}

(Received 17 April 2018; published 14 June 2018)

\begin{abstract}
Vapor condensation on weakly interacting substrates leads to the formation of three-dimensional (3D) nanoscale islands (i.e., nanostructures). While it is widely accepted that this process is driven by minimization of the total film/substrate surface and interface energy, current film-growth theory cannot fully explain the atomic-scale mechanisms and pathways by which 3D island formation and morphological evolution occurs. Here, we use kinetic Monte Carlo simulations to describe the dynamic evolution of single-island shapes during deposition of $\mathrm{Ag}$ on weakly interacting substrates. The results show that $3 \mathrm{D}$ island shapes evolve in a self-similar manner, exhibiting a constant height-to-radius aspect ratio, which is a function of the growth temperature. Furthermore, our results reveal the following chain of atomic-scale events that lead to compact 3D island shapes: 3D nuclei are first formed due to facile adatom ascent at single-layer island steps, followed by the development of sidewall facets bounding the islands, which in turn facilitates upward diffusion from the base to the top of the islands. The limiting atomic process which determines the island height, for a given number of deposited atoms, is the temperature-dependent rate at which adatoms cross from sidewall facets to the island top. The overall findings of this study provide insights into the directed growth of metal nanostructures with controlled shapes on weakly interacting substrates, including two-dimensional crystals, for use in catalytic and nanoelectronic applications.
\end{abstract}

DOI: 10.1103/PhysRevMaterials.2.063401

\section{INTRODUCTION}

Atoms deposited from the vapor phase on weakly interacting substrates self-assemble in dispersed three-dimensional (3D) nanoscale islands (i.e., nanostructures) which grow in size, coalesce, and eventually form a continuous structure that is characterized by roughness at the growth front and an average height of up to hundreds of atomic layers [1-3]. A notable example is the deposition of metal films on twodimensional (2D) crystals (e.g., graphene and $\mathrm{MoS}_{2}$ ) [4-6] for which the tendency toward the formation of 3D agglomerates imposes technological obstacles for the use of 2D materials in a wide range of switching and, in some cases, catalytic devices [4-14]. Thus, understanding atomistic mechanisms that govern 3D island formation and shape evolution is a key step toward controlling film morphology and, by extension, the functionality of devices based on weakly interacting film/substrate materials systems.

From a purely thermodynamic perspective, 3D nanostructure formation is the result of interface-energy minimization; larger adatom/adatom than adatom/substrate interface energy provides a driving force to minimize the film/substrate contact area [1,2]. However, vapor condensation and film growth proceed far from thermodynamic equilibrium and thus morphological evolution is primarily determined by the relative rates of competing atomistic structure-forming processes (i.e., by kinetics) [1-3]. Currently, the most detailed atomistic

*kostas.sarakinos@liu.se description of far-from-equilibrium 3D island formation is based on homoepitaxial systems in which 3D islands (mounds) form by deposition onto existing small islands, followed by atomic-step descent limited by the Ehlrich-Schwöbel barrier [15-19]. However, for weakly interacting film/substrate systems-including $\mathrm{Ag} / \mathrm{SiO}_{2}$ [20-24], $\mathrm{Pd} / \mathrm{TiO}_{2}$ [25], $\mathrm{Cu} / \mathrm{ZnO}$ [26,27], and Dy/graphene [4,28] —3D islands develop before the initially formed one-atom-high islands are large enough to efficiently capture vapor-phase deposition flux. Moreover, 3D island formation is also known to occur in the absence of deposition flux due to surface restructuring via dewetting [29]. These observations suggest an atomistic pathway which facilitates material transport from the base of atomic islands to their upper layer, a highly unlikely process in standard homoepitaxial thin-film growth theory due to large activation barriers associated with upward interlayer transport across step edges [30].

Following the early categorization and description of 3D (Volmer-Weber) film growth by Bauer [31], recent studies of metal-film and nanostructure growth on graphite [32], graphene [4], and other 2D materials [5,6] have explained experimentally observed growth morphologies as a function of the ratio between the adsorption energy ( $\left.E_{\text {ads }}\right)$ of metal atoms on the substrate and the bulk-metal cohesive energy $\left(E_{\mathrm{coh}}\right)$; the lower the $E_{\text {ads }} / E_{\text {coh }}$ ratio, the stronger the tendency toward 3D growth. Intuitively, this can be understood as the metal atoms being more weakly bound to the substrate, which facilitates their ascent onto the first layer of an island by either direct hopping or an exchange mechanism [33]. However, beyond the first atomic layer, growth is primarily homoepitaxial, and thus 

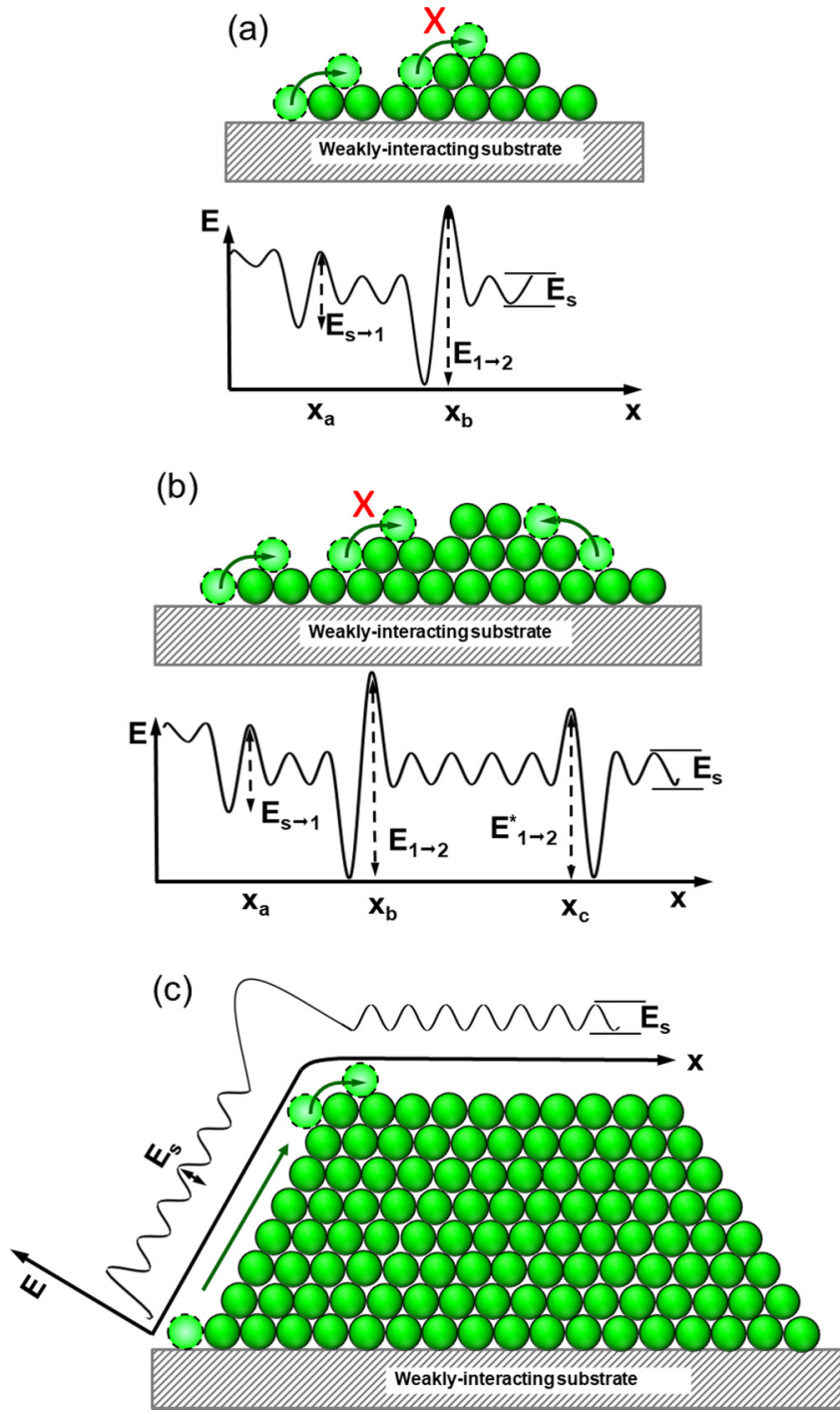

FIG. 1. Schematic illustrations of atomic structure, potential energy landscape, and upward atomic transport mechanisms during $3 \mathrm{D}$ island formation on weakly interacting substrates. (a) A weak adatom/substrate vs adatom/film interaction facilitates transport from the substrate onto the first island layer (position $x_{a}$ with barrier $E_{s \rightarrow 1}$ ), but the onset of homoepitaxial growth conditions on the first layer imposes a larger barrier for an adatom crossing the atomic step to the second island layer (position $x_{b}$ with barrier $E_{1 \rightarrow 2}>E_{s \rightarrow 1}$ ). (b) Assisted up-stepping mechanism: The close horizontal proximity of atomic steps facilitates upward atomic transport onto the second island layer (position $x_{c}$ for which the ascent barrier $E_{1 \rightarrow 2}^{*}$ is smaller than the corresponding value $E_{1 \rightarrow 2}$ at position $x_{b}$, but larger than $E_{s \rightarrow 1}$ at $x_{a}$ ). (c) The formation of low-index sidewall facets provides a facile diffusion pathway for upward mass transport with a barrier $E_{S}$ that is smaller than the step-ascent barrier $E_{1 \rightarrow 2}$ in (a) and (b). Solid spheres represent island atoms, while hollow spheres represent migrating adatoms. The diffusion direction is indicated by arrows, while " $X$ " represents an energetically unfavorable pathway.

governed once again by limited step descent and negligible step ascent. This is illustrated in Fig. 1(a), which shows a schematic representation of the atomic structure, the potential energy landscape, and the atomic-step ascent from the substrate to the first island layer (position $x_{a}$ with barrier $E_{s \rightarrow 1}$ ) and from the first to the second island layer (position $x_{b}$ with barrier $E_{1 \rightarrow 2}>E_{s \rightarrow 1}$ ). The effect of $E_{1 \rightarrow 2}$ being larger than $E_{s \rightarrow 1}$ is clearly exemplified by the heteroepitaxial $\mathrm{Co} / \mathrm{Cu}(111)$ system [34], in which Co is limited to bilayer island formation (for material coverages in the submonolayer (ML) regime) as step ascent from the first to second Co layer is more difficult than ascent from the substrate to the first layer. Thus, weak adatom/substrate bonds (manifested by low $E_{\text {ads }} / E_{\text {coh }}$ ratios) can only explain initial 3D island formation, not the way by which the island shape evolves with continued deposition.

A mechanism that can explain continued growth, beyond the second atomic layer, of 3D islands on weakly interacting substrates is assisted up-stepping [26,27,35], which assumes a reduction in the step ascent barrier, when adjacent layers are separated horizontally by only one atom, due to an attractive force from the upper step edge (see, for example, position $x_{c}$ in Fig. 1(b), for which the ascent barrier $E_{1 \rightarrow 2}^{*}$ is smaller than the corresponding value $E_{1 \rightarrow 2}$ at position $x_{b}$, but larger than $E_{s \rightarrow 1}$ at $x_{a}$ ). Modification of step-edge barriers due to short-range interactions has also been suggested to be relevant for facilitating the concerted exchange of atoms toward the upper layer of atomic islands in homoepitaxial systems [e.g., $\mathrm{Cu} / \mathrm{Cu}(111)$ and $\mathrm{Ag} / \mathrm{Ag}(100)][36,37]$. In general, the close horizontal proximity of adjacent step edges offers the possibility of accelerated interlayer mass-transport pathways [38-44], and hence may be of relevance for explaining 3D islands shape evolution far from the film/weakly interacting-substrate interface.

An alternative route to $3 \mathrm{D}$ island growth relies on the formation of low-index sidewall facets, rather than stepped structures. These facets have been suggested to facilitate growth of AlSn alloy films on weakly interacting substratesincluding amorphous- $\mathrm{C}, \mathrm{SiO}_{2}, \mathrm{NaCl}$, and mica [3,45-47]—by providing pathways for accelerated mass transport between the base and the upper layer of 3D atomic islands. Moreover, facet formation has been experimentally observed during the deposition of metal films on graphene and graphite surfaces [4,32]. Hence, in the context of 3D growth beyond the second layer, the absence of steps may allow adatoms to ascend multiple layers by encountering a terrace diffusion barrier $\left[E_{s}\right.$ in Fig. 1(c)] that is much smaller than its corresponding step-ascent counterpart $E_{1 \rightarrow 2}$ in Figs. 1(a) and 1(b)]. The final $3 \mathrm{D}$ island shape would then be controlled by the rates of atom diffusion on the facets and facet/facet crossing, rather than the rate of step-edge crossing [48-50]. For fully developed and nonsupported nanoparticles, simulations and analytical models [51,52] suggest that faceting is promoted by low adatom residence times (i.e., high chemical potentials) on nanoparticle sidewalls combined with low rates of adatom generation from kinks along the facet periphery. However, the atomistic formation of such facets from an initial island nucleus in the presence of a deposition flux has not been explained and the role of the substrate in the formation and structure of these facets has not been investigated.

In order to evaluate the relevance of the above mechanisms for 3D island formation on weakly interacting substrates and to explore the possibility of novel pathways for upward atomic transport, we develop a kinetic Monte Carlo (KMC) algorithm and perform growth simulations to probe the dynamic shape evolution of a single island. A physical model based upon a bond-counting scheme is first developed using $\mathrm{Ag} / \mathrm{Ag}(111)$ 
homoepitaxy as a starting point, chosen due to the immense experimental and theoretical literature on this film/substrate system $[41,43,53-56]$. The model is validated by showing that simulations: (i) reproduce experimentally observed trends of island morphological evolution during $\mathrm{Ag} / \mathrm{Ag}(111)$ homoepitaxy as a function of film-growth temperature [54], and (ii) yield equilibrium island shapes upon annealing, at elevated temperatures and in the absence of deposition, of hemispherical islands on weakly interacting substrates [57].

After being validated, the KMC algorithm is used to investigate Ag island growth on weakly interacting (111) substrates formed by tuning the film/substrate bond strength to be smaller than the film/film bond strength. Our results show that $3 \mathrm{D}$ island shape evolution proceeds in a self-similar manner by maintaining a constant height-to-radius aspect ratio, which is a function of the deposition temperature. The island initially contracts into a 3D shape via upward step crossing, followed by the formation of sidewall nanofacets which facilitate further upward mass transport. We also find that the rate-limiting atomic process which determines the island height is adatom crossing from sidewall facets to the island top layer.

The paper is organized as follows. Section II describes the physical model used in the simulation code. Section III presents simulation results for island growth in homoepitaxial systems, followed by results and discussion on simulations of island growth on weakly interacting substrates. The overall results are summarized in Sec. IV.

\section{THEORY AND COMPUTATIONAL DETAILS}

Our physical model describes atom-by-atom adsorption on a discrete lattice and adatom diffusion between neighboring surface sites. The starting point, as a reference for testing our model, is $\mathrm{Ag} / \mathrm{Ag}(111)$ homoepitaxial growth for which adatoms probe both fcc and hcp adsorption sites on the fcc (111) surface (Fig. 2). In addition, we apply the condition that atoms must always be supported by at least two nearestneighbor atoms, whose positions are not constrained to any particular atomistic geometry. This allows freedom of mo-

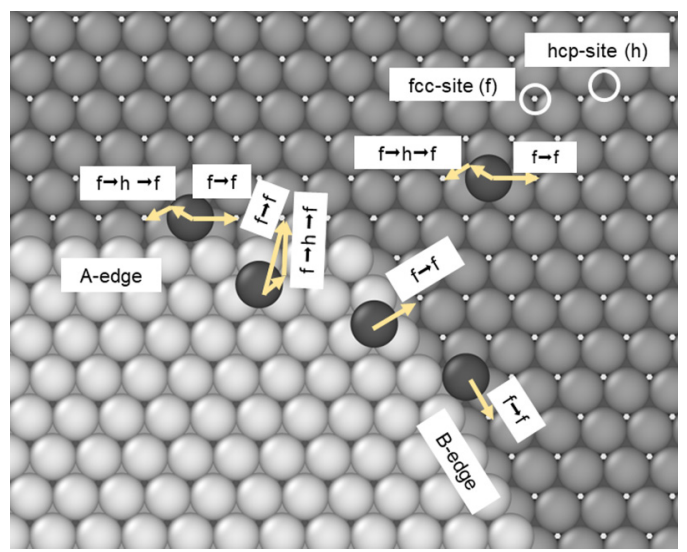

FIG. 2. Illustration of characteristic diffusion pathways provided by a refined lattice. $f \rightarrow f$ is a direct fcc-to-fcc hop, and $f \rightarrow h$ is a fcc-to-hcp hop. The diffusion directions of adatoms (black spheres) are indicated by arrows. Alternating $A$ and $B$ edges are composed of $\langle 110\rangle /\{100\}$ and $\langle 110\rangle /\{111\}$ nanofacets, respectively. tion in all three spatial dimensions and, thereby, provides the ability to treat transition-state sites. However, stable adsorption sites require a minimum of three nearest-neighbor atoms.

The explicit inclusion of hcp adsorption sites in KMC simulations has previously been used to study stacking-fault nucleation [56,58] and grain-boundary propagation [59] in $\operatorname{Ir}(111)$, and is referred to as providing a refined lattice. Here, a refined lattice is used to describe the different atomistic geometries that adatoms encounter as they diffuse along, and across, the two sets of step edges that exist on fcc(111) island surfaces - commonly referred to as $A[(100)$ nanofaceted] and $B$ [(111) nanofaceted] steps (Fig. 2) [60,61]. Along the $B$ edge, only direct hops between fcc sites $(f \rightarrow f)$ are possible as the adjacent row of hcp sites closest to the step edge are obscured by edge atoms. In contrast, adatom diffusion along the $A$ edges occur via both $f \rightarrow f$, as well as alternating fcc and hcp sites $(f \rightarrow h \rightarrow f)$. Moreover, descent from an fcc site directly above the $B$ edge to a lower fcc edge site is a direct process $(f \rightarrow f)$ involving only a transition state with two nearest-neighbor edge atoms. In contrast, adatom descent to a lower fcc site at an $A$ edge may occur either directly $(f \rightarrow f)$ or via an adjacent hcp site $(f \rightarrow h \rightarrow f)$. The differences in adatom diffusion pathways, combined with different activation barriers for $f \rightarrow f, f \rightarrow h$, and $h \rightarrow f$ hops, as described below, reproduce the well-established anisotropy in edge diffusion and step-crossing rates at the two types of fcc edges [16,36,54,60-64].

Diffusion rates $v$ in our KMC model are calculated using the Arrhenius expression,

$$
v=v_{0} \exp \left(-\frac{E_{a}}{k_{B} T}\right),
$$

in which $v_{0}$ is the attempt frequency-assumed to be $1.25 \times$ $10^{12} \mathrm{~s}^{-1}$, representing an average of the range of published experimental and theoretical $v_{0}$ values for $\mathrm{Ag}$ on $\mathrm{Ag}(111)$ terraces [54,65-69] — and $E_{a}$, the activation energy barrier of the particular diffusion step in question. $E_{a}$ values are obtained via a bond-counting scheme (BCS), which is based on the change in coordination number as an atom moves from its initial to its final adsorption site [60].

The total activation barrier $E_{a}$ in Eq. (1) consists of three parts,

$$
E_{a}=E_{k}+E_{i f}+C_{s c},
$$

in which $E_{k}$ is the kinetic barrier, $E_{i f}$ is the energy difference between initial adsorption site $i$ and final adsorption site $f$, and $C_{s c}$ is a term which is used in order to adjust $E_{a}$ in the case of step crossing. We calculate $E_{k}$ via the expression

$$
E_{k}=C_{k} \min \left\{\sum_{n} E_{D, n} N N_{n, i}, \sum_{n} E_{D, n} N N_{n, f}\right\} .
$$

In Eq. (3), $N N$ denotes the number of nearest neighbors to a given adsorption site, $E_{D}$ is the energy per coordination atom in the kinetic barrier, and the index $n$ denotes the type of species with which the adatom interacts, either substrate or film. The prefactor $C_{k}$ is used for adjusting the barrier values for the case that the diffusion process includes $f \rightarrow h$ and $h \rightarrow f$ 
TABLE I. Activation energies for selected Ag adatom migration processes on Ag(111). Symbol definitions are $t=$ terrace, $e_{A}=A$ edge, $e_{B}=B$ edge, and $c=$ corner. The migration paths used to determine BCS barriers are direct hops between fcc sites $(f \rightarrow f)$ and via a hcp site $(f \rightarrow h \rightarrow f)$. Step-edge ascent and descent barriers are calculated for an adatom starting from an initial adsorption site adjacent to an island edge and moving to a transition state, supported by two nearest-neighbors at the edge. Published experimental and theoretical activation energy reference values, as well as values from our NEB calculations, are also listed. Note that experimentally determined barrier values are averages and not specific to a particular migration path.

\begin{tabular}{|c|c|c|c|c|}
\hline \multicolumn{5}{|c|}{ Activation barriers $(\mathrm{eV})$} \\
\hline $\begin{array}{l}\text { Migration } \\
\text { path }\end{array}$ & $\begin{array}{c}\text { BCS } \\
\text { (present study) }\end{array}$ & $\begin{array}{c}\text { Literature } \\
\text { (theoretical) } \\
{[30,70]}\end{array}$ & $\begin{array}{c}\text { NEB } \\
\text { (present study) }\end{array}$ & $\begin{array}{c}\text { Literature (experimental) } \\
\text { (experimental) } \\
{[16,54,69,71-78]}\end{array}$ \\
\hline$t \rightarrow t(f \rightarrow h)$ & 0.065 & 0.063 & 0.066 & $0.05-0.18$ \\
\hline$t \rightarrow t(f \rightarrow f)$ & 0.081 & & & \\
\hline$t \rightarrow t(h \rightarrow f)$ & 0.008 & & & \\
\hline$e_{A} \rightarrow e_{A}(f \rightarrow h)$ & 0.261 & $0.258,0.275$ & 0.259 & \\
\hline$e_{A} \rightarrow e_{A}(f \rightarrow f)$ & 0.313 & & 0.259 & \\
\hline$e_{A} \rightarrow e_{A}(h \rightarrow f)$ & 0.011 & & & \\
\hline$e_{B} \rightarrow e_{B}(f \rightarrow f)$ & 0.313 & $0.302,0.310$ & 0.306 & \\
\hline$e_{A} \rightarrow c(f \rightarrow h)$ & 0.338 & & 0.327 & \\
\hline$e_{B} \rightarrow c(f \rightarrow f)$ & 0.361 & 0.360 & 0.385 & \\
\hline$c \rightarrow e_{A}(f \rightarrow h)$ & 0.087 & 0.072 & 0.065 & \\
\hline$c \rightarrow e_{B}(f \rightarrow f)$ & 0.109 & 0.098 & 0.109 & \\
\hline Step descent $(A: h \rightarrow f)$ & 0.284 & 0.333 & 0.436 & $0.22,0.23$ \\
\hline Step descent $(A: f \rightarrow f)$ & 0.333 & 0.348 & 0.476 & \\
\hline Step descent $(B: f \rightarrow f)$ & 0.322 & 0.332 & 0.428 & \\
\hline Step ascent $(A)$ & 0.836 & 0.821 & 1.066 & \\
\hline Step ascent $(B)$ & 0.825 & 0.825 & 1.073 & \\
\hline (001) terrace diffusion & 0.452 & 0.448 & 0.456 & $0.33-0.45$ \\
\hline
\end{tabular}

transitions [57]. In particular, we explicitly choose $C_{k}^{h \rightarrow f}=$ 0.1. This renders hcp sites on the $\operatorname{Ag}(111)$ surface metastable, which is consistent with scanning tunneling microscopy (STM) studies reporting a low nucleation probability for Ag hcp islands on $\operatorname{Ag}(111)$ surfaces [54].

The $E_{i f}$ term in Eq. (2) is expressed as

$$
E_{i f}=\max \left\{\sum_{n} E_{B, n} r n d\left(N N_{n, i}-N N_{n, f}+0.5\left(N N N_{n, i}-N N N_{n, f}\right)\right), 0\right\} .
$$

$E_{B}$ in Eq. (4) is the pairwise bond strength between adatoms and film, and/or substrate, species. Next-nearest neighbors $(N N N)$, in addition to $N N$, are also included when calculating $E_{i f}$ in order to account for both fcc and hcp lattices [57]. The scaling factor 0.5 for all terms containing $N N N$ is determined by approximating the interaction strength between atom pairs as scaling with their separation distance $r$ as $1 / r^{2}$. Thus, for a $N N N$ at a distance $r=\sqrt{2} r_{0}$ on the fcc(111) surface, in which $r_{0}$ is the separation between $N N$, the interaction strength, and hence, the potential energy, is reduced by half. The rounding function ( $r n d$ ) has been introduced to Eq. (4), since we found that it allows one to achieve good agreement between BCS barrier values and reference values (see the next paragraph) for the majority of the studied processes [57]. For cases where this was not possible, including diffusion along the two types of step edges, all processes involving kinks, and diffusion along fcc(100) facets on 3D islands, the additive term $C_{i f}$ is used to adjust $E_{a}$ values.
Equations (2) through (4) include a total of eight parameters which are uniquely determined (see Supplemental Material [57] for values of these parameters) to reproduce published theoretical barriers for $\mathrm{Ag} / \mathrm{Ag}(111)$ [30,70]. Moreover, BCS values are also compared to experimental results [16,54,69,71$78]$, as well as barriers obtained from our nudged-elasticband (NEB) calculations using an embedded atom method interatomic potential [79]. Activation barriers for selected processes are listed in Table I. The results show that our BCS predictions are in good agreement with reference values. In particular, BCS correctly yields an average barrier for adatom diffusion along $A$ edges $\left(e_{A} \rightarrow e_{A}\right)$ that is smaller than along $B$ edges $\left(e_{B} \rightarrow e_{B}\right)$. This, combined with the fact that both hcp and fcc adsorption sites are available along the $A$ edge (cf. Fig. 2), enhances $A$-edge, compared to $B$-edge, diffusion rates in agreement with literature results $[54,60]$. The well-established corner-crossing asymmetry at $\mathrm{Ag} / \mathrm{Ag}(111)$ islands that favors crossing from $B$ to $A$ edges [54,60] is also 
reproduced by our BCS calculations. Finally, the BCS results correctly capture the known preference for $B$-step descent [54,60-64]. Step crossing at $A$ edges is less likely due to the presence of alternating hcp sites (see Fig. 2) which impose an additional jump to complete the down-stepping process. We also used our BCS formalism to determine the potential-energy landscape and, thereby, calculate the relative energy difference between characteristic fcc adsorption sites, which are listed in Supplemental Material [57].

To model a weakly interacting film/substrate system, we use

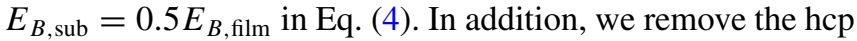
sites at the substrate surface in order to allow isotropic adatom diffusion along island edges to mimic an amorphous substrate. Beyond the first atomic layer, the full fcc crystal structure, as well as homoepitaxial growth kinetics, are always applied. The reduction of $E_{B \text {,sub }}$, for describing weak film/substrate interactions, results in a step-ascent barrier of $0.21 \mathrm{eV}$ (compared to $\sim 0.82 \mathrm{eV}$ for homoepitaxy; see Table I). This shows that our BCS approach is capable of yielding kinetic conditions that allow upward mass transport at elevated growth temperatures.

Simulations are performed using the standard KMC iteration algorithm, which is readily available in textbooks [80], and hence not described here. Atoms are not allowed to overlap, which means hcp (fcc) sites adjacent to an occupied fcc (hcp) site are unavailable. All simulations within the present study are performed in a cubic simulation box with sides of length 100 atoms [approximately $29 \mathrm{~nm}$ for $\mathrm{Ag}(111)$ ]; periodic boundary conditions are applied along orthogonal in-plane surface directions. Growth simulations are carried out, for both homoepitaxial and weakly interacting substrates, at temperatures $T_{s}$ between 100 and $500 \mathrm{~K}$ for coverages $\Theta$ up to $1 \mathrm{ML}$, with a deposition rate of $10 \mathrm{ML} / \mathrm{s}$. The OVITO software package [81] is used to visualize island formation and shape evolution, in steps of $10^{-4}$ to $10^{-2} \mathrm{ML}$, throughout all growth stages.

\section{RESULTS AND DISCUSSION}

In order to validate the physical model described in the previous section, the simulation code is first used to replicate 2D $\mathrm{Ag} / \mathrm{Ag}(111)$ island shapes as a function of $T_{s}$. Figure 3 shows simulated islands after $0.5 \mathrm{ML}$ of homoepitaxial $\mathrm{Ag}$ growth on $\operatorname{Ag}(111)$ at $T_{s}$ values ranging from 100 to $450 \mathrm{~K}$.

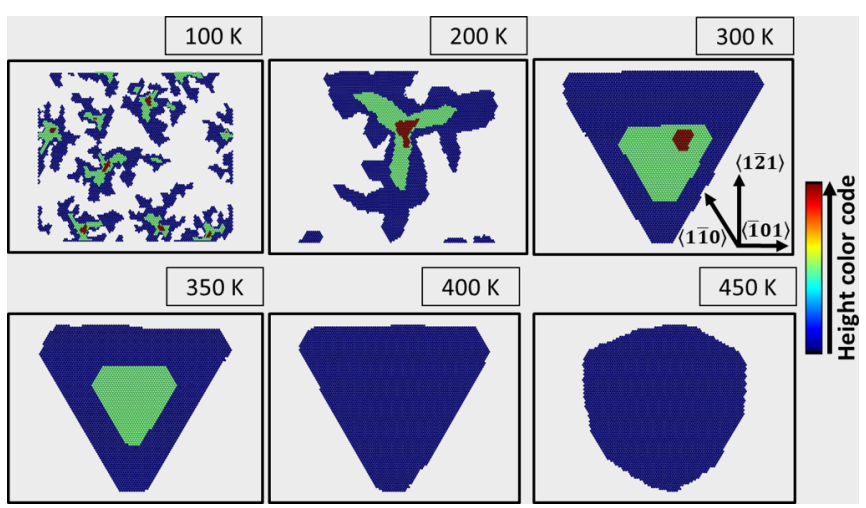

FIG. 3. Simulated island morphologies at $\Theta=0.5$ ML during homoepitaxial $\mathrm{Ag}$ growth on $\mathrm{Ag}(111)$ at $T_{s}$ values ranging from 100 to $450 \mathrm{~K}$.
At $100 \mathrm{~K}$, multiple islands are obtained, while growth at all larger $T_{s}$ values yields single islands. These results show that the predictions of our KMC algorithm are consistent with experimental observations [54]; low adatom surface diffusion rates (due to low $T_{s}$ ) lead to enhanced nucleation probabilities, shorter nucleation lengths, and higher island densities. At 100 and $200 \mathrm{~K}$, the islands grow by diffusion-limited aggregation (DLA), displaying dendritic shapes with preferred growth of $B$ edges (especially at $200 \mathrm{~K}$ ).

Increasing $T_{s}$ to $300-400 \mathrm{~K}$ yields more compact, nearly triangular islands preferentially bounded by $B$ edges. This indicates that island shape evolution proceeds, in agreement with experimental and theoretical results [54,82], under conditions of strong corner-crossing anisotropy favoring edge crossing from $B$ to $A$, which allows $A$ edges to grow faster than $B$ edges and, hence, shrink in size. Additional step-edge growth simulations have shown that $A$ edges grow considerably rougher than $B$ edges, which further validates the effective implementation of the corner-crossing asymmetry in our KMC model [83]. Moreover, the smoothening of island $B$ edges, leading to compact islands with increasing growth temperature, is consistent with $\mathrm{Ag} / \mathrm{Ag}$ (111) homoepitaxial data [53,54] and shows that our model correctly treats adatom diffusion at the island periphery.

At $450 \mathrm{~K}$, which is $\sim 35 \%$ of the melting temperature of bulk Ag, the island has a nearly hexagonal shape indicating the onset of edge-atom detachment. This process generates kinks along both edges, which decreases their chemical potential and, hence, the net flux of atoms from $B$ toward $A$ edges. This, in turn, yields an island shape closer to equilibrium, i.e., an island that is not preferentially bounded by a specific edge, but rather exhibits a structure determined by the hexagonal symmetry of the fcc(111) substrate surface. These results are consistent with experimental data for several fcc(111) homoepitaxial and heteroepitaxial systems $[60,63]$.

Figure 3 also shows enhanced downward interlayer transport with increasing temperature. While we typically obtain 2-3 layers at $T_{s}=100-300 \mathrm{~K}$, islands grown at 400 and $450 \mathrm{~K}$ are single layers. This trend is also in agreement with experimental observations and theoretical predictions regarding upper-layer nucleation and mound formation [60]. Thus, key features of our KMC algorithm are validated by the homoepitaxial-growth literature.

Besides $\mathrm{Ag}$ on $\mathrm{Ag}(111)$ homoepitaxy, we also performed annealing simulations of hemispherical islands on weakly interacting substrates, which yielded island shapes that are consistent with the equilibrium Winterbottom construction crystal shape for $\mathrm{Ag}$ [57]. This provides further validation of our $\mathrm{KMC}$ model, which is used in the following to investigate shape evolution of single 3D Ag islands.

Figure 4 presents simulated island morphologies on a weakly interacting substrate at $T_{s}=100$ to $500 \mathrm{~K}$ for the same coverage, $\Theta=0.5 \mathrm{ML}$, as in Fig. 3. Consistent with the homoepitaxial results in Fig. 3, growth on weakly interacting substrates at the lowest $T_{s}$ value $(100 \mathrm{~K})$ results in multiple islands which exhibit dendritic shapes characteristic of DLA growth. At all other growth temperatures in Fig. 4, only one island is formed, which shows that our simulation algorithm reproduces, even for growth on weakly interacting substrates, the physically expected dynamic competition between adatom 


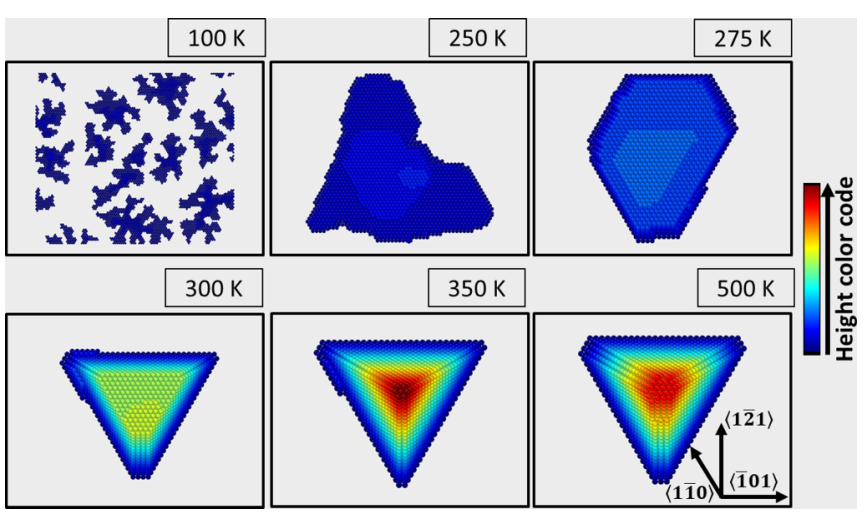

FIG. 4. Simulated island morphologies during growth of Ag on weakly interacting substrates at $T_{s}$ values ranging from 100 to $500 \mathrm{~K}$. Each panel corresponds to a total deposition of $\Theta=0.5 \mathrm{ML}$.

diffusion and island nucleation. At $250 \mathrm{~K}$, the island periphery is rough, primarily bounded by edges with (100) nanofacets, and consists of five atomic layers.

A further increase in $T_{s}$ to $275 \mathrm{~K}$ results in a smoother island periphery and the formation of (111) edge nanofacets whose total length is approximately equal to that of the (100) edge facets. In addition, layer-by-layer growth is observed on both types of side nanofacets. Moreover, the island height increases, compared to those at lower $T_{s}$, to seven atomic layers. Similar island morphologies have been experimentally reported in STM studies of $\mathrm{Pb}$ and $\mathrm{Dy}$ growth on graphene [4].

Dramatic changes in island morphology are observed with $T_{s} \geqslant 300 \mathrm{~K}$. The (100) edge facets largely vanish, the islands are now bounded almost entirely by (111) sidewall facets, and the islands continue to grow vertically. Island heights reach 16,27 , and 24 layers with $T_{s}$ equal to 300,350 , and $500 \mathrm{~K}$, respectively.

In order to quantify the effect of $T_{s}$ on island morphological evolution, we determined the island height-to-radius ratios $h / r$ at each $T_{s}$ value as a function of the coverage $\Theta$. The height $h$ corresponds to the total number of atomic layers on the island, while the radius $r$ (in $\AA$ ) is taken to be equal to the radius of a circle that has the same area as the initial island layer. Figure 5(a) presents $h / r$ curves as a function of $\Theta$ for the $T_{s}$ values in Fig. 4. At all temperatures, the $h / r$ ratio exhibits a sharp increase during the very early stages of island formation $\left(\Theta<5 \times 10^{-3} \mathrm{ML}\right)$. Then, depending on $T_{s}, h / r$ either immediately reaches $\left(T_{s} \geqslant 300 \mathrm{~K}\right)$, or decreases $\left(T_{s} \leqslant 275 \mathrm{~K}\right)$ toward, a temperature-dependent steady-state value $\left.\frac{h}{r}\right|_{s s}$, indicating that the 3D island shapes evolve in a self-similar manner. Moreover, all $h / r$ vs $\Theta$ curves for $T_{s} \geqslant 300 \mathrm{~K}$ and $\Theta \gtrsim 0.1 \mathrm{ML}$ exhibit regions of coverage $\Theta$ in which $h / r$ decreases with a relatively small slope, separated by extremely narrow regions of $\Theta$ in which $h / r$ increases abruptly. This is highlighted in Fig. 5(b), which shows a higher resolution image of $h / r$ vs $\Theta$ at $T_{s}=300 \mathrm{~K}$ with $\Theta$ ranging from 0.10 to 0.28 ML. The connection between the oscillatory behavior of the $h / r$ ratio and $3 \mathrm{D}$ island formation evolution is discussed below.

Figure 5(c) presents a plot of $\left.\frac{h}{r}\right|_{s s}$ values a function of $\left.T_{s} \cdot \frac{h}{r}\right|_{s s}$ is approximately constant at $\sim 0.15$ over the $T_{s}$ range from 100 to $250 \mathrm{~K}$ and increases sharply at $T_{s}$ between 250 and $325 \mathrm{~K}$ to reach a value $\sim 1.9$ with $T_{s}>325 \mathrm{~K}$.
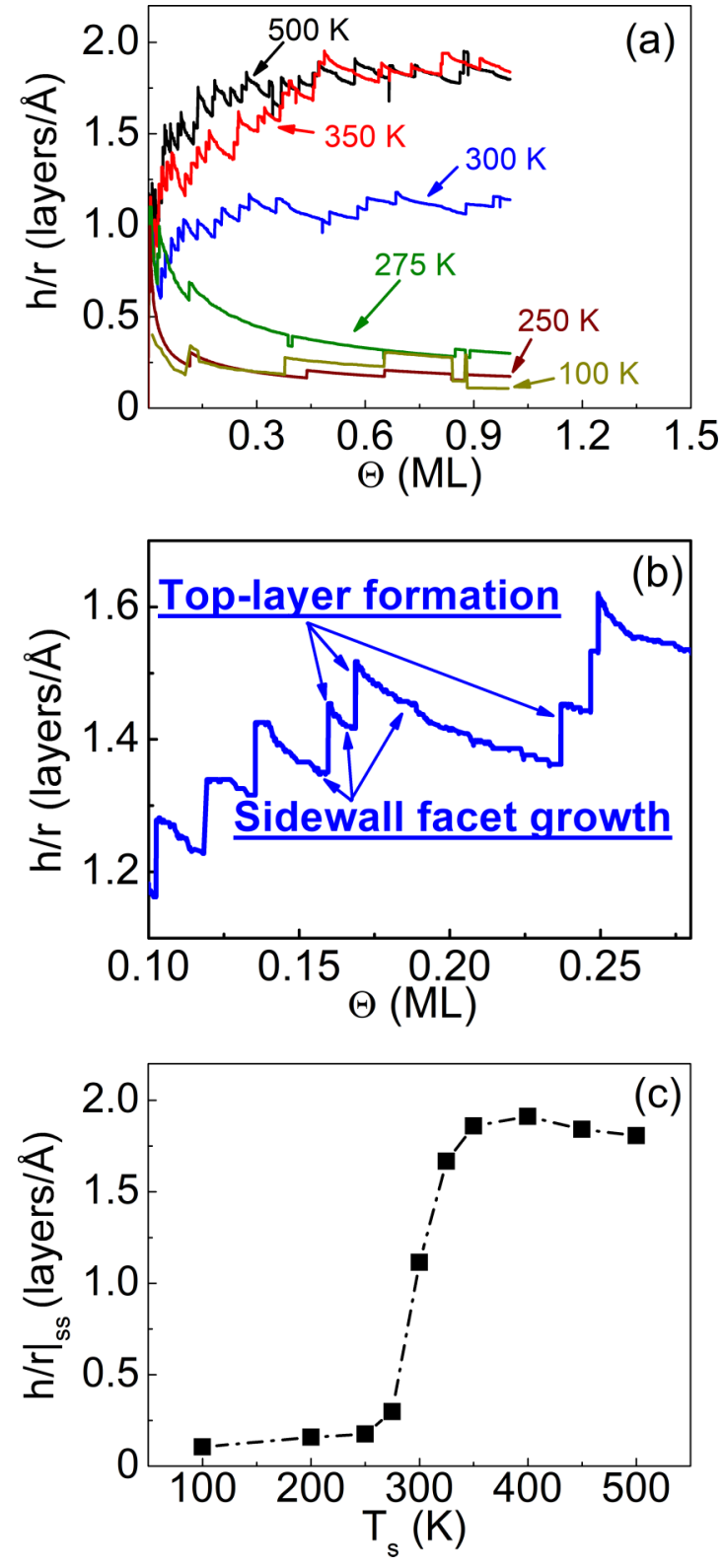

FIG. 5. (a) Height-to-radius $h / r$ ratios of simulated Ag islands deposited on weakly interacting substrates as a function of coverage $\Theta$ at $T_{s}$ values ranging from 100 to $500 \mathrm{~K}$. (b) Higher-resolution image of $h / r$ vs $\Theta$ at $T_{s}=300 \mathrm{~K}$ highlighting island growth stages as noted (see text for further details). (c) Steady-state $h / r$ values $\left.\frac{h}{r}\right|_{s s}$ as a function of $T_{s}$. The dashed/dotted line is a guide for the eye.

To understand the atomistic origin of the trends observed in Figs. 4 and 5, we investigated the initial stages of island shape evolution at coverages up to $\Theta=2.5 \times 10^{-2} \mathrm{ML}$ with a resolution of $\sim 10^{-4} \mathrm{ML}$. Representative island images from simulations performed at $T_{s}=350 \mathrm{~K}$ are shown in Fig. 6 . $3 \mathrm{D}$ island formation already occurs at $\Theta=5.0 \times 10^{-4} \mathrm{ML}$. At this coverage, the initial island layer contains only a few atoms and hence direct deposition of adatoms on the island is highly unlikely. Instead, second-layer nucleation is primarily driven by adatoms which ascend the single-layer island from the substrate surface. This process is facilitated by the weak film/substrate bond strength. At a slightly larger coverage, 


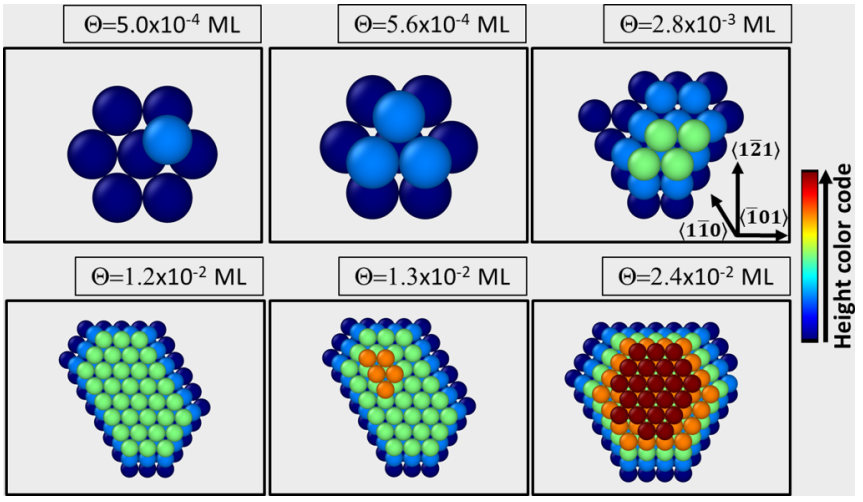

FIG. 6. Simulated shape evolution during initial Ag island growth, with coverages $\Theta$ ranging from $5 \times 10^{-4}$ to $2.4 \times 10^{-2} \mathrm{ML}$, on a weakly interacting substrate at $T_{s}=350 \mathrm{~K}$.

$\Theta=5.6 \times 10^{-4} \mathrm{ML}$, atoms ascending onto the first layer cause the second layer to grow laterally in plane until it reaches the island edge and forms small sidewall nanofacets, which offer a pathway for upward adatom diffusion from the substrate beyond the second layer. The third layer does not form until a coverage of $\Theta=2.8 \times 10^{-3} \mathrm{ML}$, at which point the second layer has expanded in plane and reached a critical radius to allow for two upward migrating adatoms to simultaneously populate, and nucleate, the third layer.

The cycle of in-plane island expansion and subsequent out-of-plane growth is repeated as more atoms are deposited, leading to formation of the fourth $\left(\Theta=1.3 \times 10^{-2} \mathrm{ML}\right)$ and fifth $\left(\Theta=2.4 \times 10^{-2} \mathrm{ML}\right)$ layer. Similar growth sequences occur at later growth stages and are the reason for the oscillatory behavior in the $h / r$ vs $\Theta$ curves for $T_{s} \geqslant 300 \mathrm{~K}$ in Figs. 5(a) and 5(b); the relatively slow periodic decrease in $h / r$ is caused by layer formation and growth on sidewall facets until the point that a new top layer is formed, resulting in an abrupt rapid increase in $h / r$ as indicated in Fig. 5(b).

A distinctly different $h / r$ vs $\Theta$ behavior is observed with $T_{s} \leqslant 275 \mathrm{~K}$. To demonstrate the reason for these differences, Fig. 7 shows the island shape evolution at $T_{s}=250 \mathrm{~K}$ for the same $\Theta$ values as in Fig. 6. At the earliest stages, $\Theta=$ $5.0 \times 10^{-4}$ and $5.6 \times 10^{-4} \mathrm{ML}$, the islands are similar to

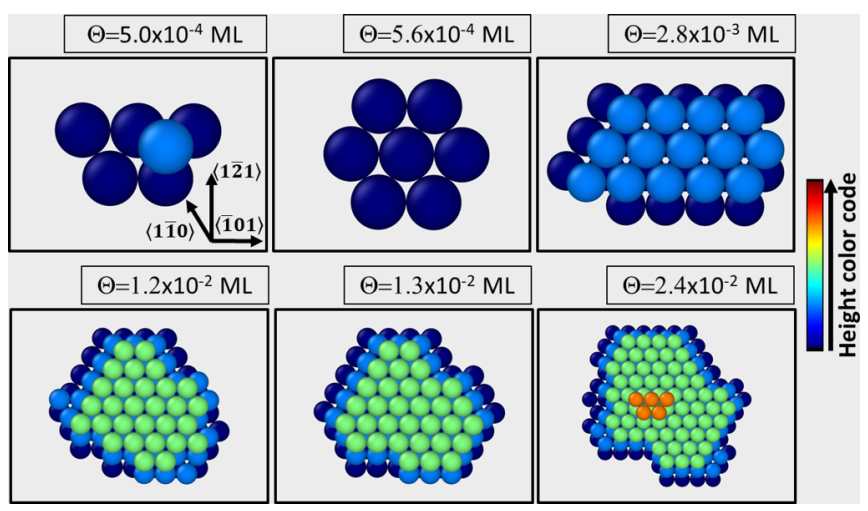

FIG. 7. Simulated shape evolution during initial $\mathrm{Ag}$ island growth, with coverages $\Theta$ ranging from $5 \times 10^{-4}$ to $2.4 \times 10^{-2} \mathrm{ML}$, on a weakly interacting substrate at $T_{s}=250 \mathrm{~K}$. those in Fig. 6; i.e., initial 3D island formation proceeds via ascent at single-layer island edges. At larger coverages, (111) sidewall nanofacets again form, but the island exhibits a much stronger preference toward in-plane growth: at $\Theta=2.4 \times$ $10^{-2} \mathrm{ML}$ and $T_{s}=250 \mathrm{~K}$, the island has a radius of $\sim 14.9 \AA$ and consists of three complete layers and an incomplete fourth layer. For comparison, at the same coverage with $T_{s}=350 \mathrm{~K}$, the island comprises five complete layers and has a smaller radius, $\sim 12.9 \AA$. In addition, the island edges are smoother at higher $T_{s}$ values, in agreement with the results shown in Fig. 4.

The results in Fig. $6\left(T_{s}=350 \mathrm{~K}\right)$ and Fig. $7\left(T_{s}=250 \mathrm{~K}\right)$ show that sidewall nanofacets form at both deposition temperatures and the islands remain faceted as new atomic layers nucleate and grow on the island top. This means that adatoms detach from the substrate and ascend onto the island at rates that are sufficiently large to allow for constant outward growth of all atomic layers and, hence, allow for the facet structure to be maintained. The rate-limiting step that determines the 3D island height is adatom crossing from the sidewall facets to the island upper layer. Increasing the growth temperature from 250 to $350 \mathrm{~K}$, increases the crossing rate and, hence, the flux of adatoms from the sidewall facet to the upper island layer. This, in turn, leads to larger top-layer nucleation rates and promotes 3D growth, as demonstrated by the transition in $\left.\frac{h}{r}\right|_{s s}$ from $\sim 0.15$ at $T_{s}<275 \mathrm{~K}$ to $\sim 1.9$ at $T_{s}>325 \mathrm{~K}$ [see Fig. 5(c)]. The tendency toward in-plane growth at $T_{s} \leqslant 275 \mathrm{~K}$ also explains the decay of the corresponding $h / r$ vs $\Theta$ curves in Fig. 5(a) following their initial sharp increase caused by second-layer nucleation.

\section{SUMMARY AND OUTLOOK}

We investigated the atomic-scale mechanisms governing 3D island growth on weakly interacting substrates, with the goal of developing an understanding of the mechanisms and atomic pathways which control film morphological evolution, and, by extension, the functionality of devices based on weakly interacting film/substrate materials systems. This was achieved by developing a KMC algorithm based on a bond-counting scheme which was validated for Ag/Ag (111) homoepitaxy and annealing of hemispherical islands on weakly interacting substrates. We then used our algorithm to probe the dynamic evolution of Ag islands on a weakly interacting (111) substrate over the temperature range $T_{s}$ from 100 to $500 \mathrm{~K}$ with a deposition rate of $10 \mathrm{ML} / \mathrm{s}$. The simulation results revealed that the 3D island height-to-radius $h / r$ aspect ratio initially increases and eventually saturates at a steady-state value $\left.\frac{h}{r}\right|_{s s}$, indicating a self-similar shape evolution which is a function of growth temperature. Islands are short and rather flat at $T_{s} \leqslant 250 \mathrm{~K}$, exhibiting $\left.\frac{h}{r}\right|_{s s}$ values of $\sim 0.15$, which sharply increase over the $T_{s}$ range 275 to $325 \mathrm{~K}$, reaching values of $\sim 1.9$ with $T_{s} \geqslant 350 \mathrm{~K}$.

Analyses of island shape evolution showed that 3D nuclei are already formed at coverages $\sim 5.0 \times 10^{-4} \mathrm{ML}$ at all $T_{s}$ values from 100 to $500 \mathrm{~K}$ due to facile adatom ascent at single-layer island steps. This is followed by the formation of sidewall nanofacets, which facilitate upward diffusion from the base to top island layer. The kinetics of these processes are different, leading to pronounced and compact 3D island growth with $T_{s} \geqslant 325 \mathrm{~K}$ and enhanced in-plane growth with 
$T_{s} \leqslant 275 \mathrm{~K}$. The primary atomic process which determines the island height, for the same number of deposited atoms, is the temperature-dependent rate at which adatoms cross from a sidewall facet to the island upper layer. The overall island shape evolution is in good agreement with experimental results for 3D metal islands grown on weakly interacting substrates, including graphene, $\mathrm{Mo}_{2} \mathrm{~S}$, and polar oxides. Moreover, the atomic-scale processes highlighted and the methodology presented in this investigation may be of relevance for studying the kinetics of island formation in strongly interacting film/substrate systems for which 3D morphology has been observed, including $\mathrm{Pb} / \mathrm{Si}(111)$ [84-86] and $\mathrm{Ag} / \mathrm{Si}(111)$ [87].

We hope that the insights generated in this investigation will trigger experimental and theoretical research for developing strategies to selectively manipulate rate-limiting atomic processes that govern shape evolution of 3D nanostructures by, e.g., use of surfactants $[88,89]$ or temporally modulated vapor fluxes [60]. Such approaches may, for example, be relevant for achieving $2 \mathrm{D}$ metal film growth on $2 \mathrm{D}$ crystals, including graphene and $\mathrm{Mo}_{2} \mathrm{~S}$, and thereby synthesize low-resistivity electrical contacts on nanoelectronic devices $[4,5,7-14]$ or fabricate catalytic devices that exhibit enhanced turnover frequencies [6].

\section{ACKNOWLEDGMENTS}

K.S. acknowledges Linköping University ("LiU Research Fellows Program, 2011-2015" and "LiU Career Contract No. Dnr-LiU-2015-01510, 2015-2020") and the Swedish Research Council (Contracts No. VR-2011-5312 and No. VR-201504630) for financial support. J.G. acknowledges financial support from the Swedish Research Council (Contract No. VR2014-5790) and the Knut and Alice Wallenberg Foundation (Contract No. KAW 2011-0094). Simulations were performed using supercomputer resources provided by the Swedish $\mathrm{Na}$ tional Infrastructure for Computing (SNIC) at the National Supercomputer Centre (NSC).
[1] M. Ohring, Materials Science of Thin Films (Academic Press, San Diego, 1991), pp. 357-360.

[2] J. E. Greene, Thin Film Nucleation, Growth, and Microstructural Evolution: An Atomistic Scale View, In Handbook of Deposition Technologies for Films and Coatings, edited by P. M. Martin (Elsevier, Amsterdam, 2005), pp. 554-558.

[3] I. Petrov, P. B. Barna, L. Hultman, and J. E. Greene, Microstructural evolution during film growth, J. Vac. Sci. Technol. A 21, S117 (2003).

[4] X. Liu, Y. Han, J. W. Evans, A. K. Engstfeld, R. J. Behm, M. C. Tringides, M. Hupalo, H. Q. Lin, L. Huang et al., Growth morphology and properties of metals on graphene, Prog. Surf. Sci. 90, 397 (2015).

[5] C. Gong, C. Huang, J. Miller, L. Cheng, Y. Hao, D. Cobden, J. Kim, R. S. Ruoff, R. M. Wallace, K. Cho et al., Metal contacts on physical vapor deposited monolayer $\mathrm{MoS}_{2}, \operatorname{ACS}$ Nano 7, 11350 (2013).

[6] W. A. Saidi, Trends in the adsorption and growth morphology of metals on the $\mathrm{MoS}_{2}$ (001) surface, Cryst. Growth Des. 15, 3190 (2015).

[7] Y. Guo, Y. Han, J. Li, A. Xiang, X. Wei, S. Gao, and Q. Chen, Study on the resistance distribution at the contact between molybdenum disulfide and metal, ACS Nano 8, 7771 (2014).

[8] S. McDonnell, R. Addou, C. Buie, R. M. Wallace, and C. L. Hinkle, Defect-dominated doping and contact resistance in $\mathrm{MoS}_{2}$, ACS Nano 8, 2880 (2014).

[9] C. Gong, S. McDonnell, X. Qin, A. Azcatl, H. Dong, Y. J. Chabal, K. Cho, and R. M. Wallace, Realistic metal-graphene contact structures, ACS Nano 8, 642 (2014).

[10] W. S. Leong, H. Gong, and J. T. L. Thong, Low-contactresistance graphene devices with nickel-etched-graphene contacts, ACS Nano 8, 994 (2014).

[11] W. Liu, D. Sarkar, J. Kang, W. Cao, and K. Banerjee, Impact of contact on the operation and performance of back-gated monolayer $\mathrm{MoS}_{2}$ field-effect-transistors, ACS Nano 9, 7904 (2015).
[12] C. Kim, I. Moon, D. Lee, M. S. Choi, F. Ahmed, S. Nam, Y. Cho, H.-J. Shin, S. Park, and W. J. Yoo, Fermi level pinning at electrical metal contacts of monolayer molybdenum dichalcogenides, ACS Nano 11, 1588 (2017).

[13] Y. Xu, C. Cheng, S. Du, J. Yang, B. Yu, J. Luo, W. Yin, E. Li, S. Dong, P. Ye et al., Contacts between two- and three-dimensional materials: Ohmic, Schottky, and $p-n$ heterojunctions, ACS Nano 10, 4895 (2016).

[14] W. S. Leong, X. Luo, Y. Li, H. K. Khoo, S. Y. Quek, and J. T. L. Thong, Low resistance metal contacts to $\mathrm{MoS}_{2}$ Devices with nickel-etched-graphene electrodes, ACS Nano 9, 869 (2015).

[15] J. Tersoff, A. W. Denier van derGon, and R. M. Tromp, Critical Island Size for Layer-By-Layer Growth, Phys. Rev. Lett. 72, 266 (1994).

[16] K. Bromann, H. Brune, H. Röder, and K. Kern, Interlayer Mass Transport in Homoepitaxial and Heteroepitaxial Metal Growth, Phys. Rev. Lett. 75, 677 (1995).

[17] J. Krug, P. Politi, and T. Michely, Island nucleation in the presence of step-edge barriers: Theory and applications, Phys. Rev. B 61, 14037 (2000).

[18] M. Li and J. W. Evans, Theoretical Analysis of Mound Slope Selection During Unstable Multilayer Growth, Phys. Rev. Lett. 95, 256101 (2005).

[19] D. Gall, S. Kodambaka, M. A. Wall, I. Petrov, and J. E. Greene, Pathways of atomistic processes on TiN(001) and (111) surfaces during film growth: An ab initio study, J. Appl. Phys. 93, 9086 (2003).

[20] E. Byon, T. W. H. Oates, and A. Anders, Coalescence of nanometer silver islands on oxides grown by filtered cathodic arc deposition, Appl. Phys. Lett. 82, 1634 (2003).

[21] H. C. Kim, T. L. Alford, and D. R. Allee, Thickness dependence on the thermal stability of silver thin films, Appl. Phys. Lett. 81, 4287 (2002).

[22] J. M. Warrender and M. J. Aziz, Effect of deposition rate on morphology evolution of metal-on-insulator films grown by pulsed laser deposition, Phys. Rev. B 76, 045414 (2007). 
[23] V. Elofsson, B. Lü, D. Magnfält, E. P. Münger, and K. Sarakinos, Unravelling the physical mechanisms that determine microstructural evolution of ultrathin Volmer-Weber films, J. Appl. Phys. 116, 044302 (2014).

[24] H. Krishna, R. Sachan, J. Strader, C. Favazza, M. Khenner, and R. Kalyanaraman, Thickness-dependent spontaneous dewetting morphology of ultrathin Ag films, Nanotechnology 21, 155601 (2010).

[25] M. J. J. Jak, C. Konstapel, A. van Kreuningen, J. Verhoeven, and J. W. M. Frenken, Scanning tunnelling microscopy study of the growth of small palladium particles on $\mathrm{TiO}_{2}$ (110), Surf. Sci. 457, 295 (2000).

[26] K. H. Ernst, A. Ludviksson, R. Zhang, J. Yoshihara, and C. T. Campbell, Growth model for metal films on oxide surfaces: $\mathrm{Cu}$ on ZnO(0001)-O, Phys. Rev. B 47, 13782 (1993).

[27] J. Yoshihara, S. C. Parker, and C. T. Campbell, Island growth kinetics during vapor deposition of $\mathrm{Cu}$ onto the $\mathrm{Zn}$-terminated ZnO(0001) surface, Surf. Sci. 439, 153 (1999).

[28] M. T. Hershberger, M. Hupalo, P. A. Thiel, and M. C. Tringides, Growth of fcc(111) Dy multi-height islands on $6 \mathrm{H}-\mathrm{SiC}(0001)$ graphene, J. Phys.: Condens. Matter 25, 225005 (2013).

[29] N. Kalfagiannis, A. Siozios, D. V. Bellas, D. Toliopoulos, L. Bowen, N. Pliatsikas, W. M. Cranton, C. Kosmidis, D. C. Koutsogeorgis, E. Lidorikis et al., Selective modification of nanoparticle arrays by laser-induced self-assembly (Mona-Lisa): Putting control into bottom-up plasmonicnanostructuring, Nanoscale 8, 8236 (2016).

[30] S. Y. Kim, I. H. Lee, and S. Jun, Transition-pathway models of atomic diffusion on fcc metal surfaces. II. Stepped surfaces, Phys. Rev. B 76, 245408 (2007).

[31] E. Bauer, Phenomenologische theorie der kristallabscheidung an oberflächen. I.Z. Kristallogr. 110, 372 (1958).

[32] D. Appy, H. Lei, C.-Z. Want, M. C. Trignides, D.-J. Liu, J. W. Ewans, and P. A. Thiel, Transition metals on the (0001) surface of graphite: Fundamental aspects of adsorption, diffusion, and morphology, Prog. Surf. Sci. 89, 219 (2014).

[33] Y. Han, B. Unal, F. Qin, D. Jing, C. J. Jenks, D.-J. Liu, P. A. Thiel, and J. W. Evans, Kinetics of Facile Bilayer Island Formation at Low Temperature: Ag/NiAl(110), Phys. Rev. Lett. 100, 116105 (2008).

[34] N. N. Negulyaev, V. S. Stepanyuk, P. Bruno, L. Diekhöner, P. Wahl, and K. Kern, Bilayer growth of nanoscale Co islands on Cu(111), Phys. Rev. B 77, 125437 (2008).

[35] C. T. Campbell, Metal films and particles on oxide surfaces: Structural, electronic and chemisorptive properties, J. Chem. Soc. Faraday Trans. 92, 1435 (1996).

[36] J. G. Amar and F. Family, Critical Cluster Size: Island Morphology and Size Distribution in Submonolayer Epitaxial Growth, Phys. Rev. Lett. 74, 2066 (1995).

[37] Y. Shim and J. G. Amar, Ab initio study of edge smoothing, atom attraction, and downward funneling in $\mathrm{Ag} / \mathrm{Ag}(100)$, Phys. Rev. B 83, 245419 (2011)

[38] X.-F. Gong, L. Wang, X.-J. Ning, and J. Zhuang, Decay mechanism of double-layer $\mathrm{Cu}$ islands on $\mathrm{Cu}(111)$, Surf. Sci. 553, 181 (2004).

[39] X.-F. Gong, B. Hu, X.-J. Ning, and J. Zhuang, Decay mechanism of double-layer islands on close-packed surfaces: Silver on $\mathrm{Ag}(111)$ and copper on $\mathrm{Cu}(111)$, Thin Solid Films 493, 146 (2005).
[40] M. Larsson, Kinetic Monte Carlo simulations of adatom island decay on $\mathrm{Cu}$ (111), Phys. Rev. B 64, 115428 (2001).

[41] K. Morgenstern, G. Rosenfeld, G. Comsa, M. R. Sørensen, B. Hammer, E. Lægsgaard, and F. Besenbacher, Kinetics of fast island decay on $\operatorname{Ag}$ (111), Phys. Rev. B 63, 045412 (2001).

[42] P. J. Feibelman, Accelerated mound decay at adjacent kinks on Cu(111), Surf. Sci. 478, L349 (2001).

[43] K. Morgenstern, G. Rosenfeld, and G. Comsa, Decay of twodimensional Ag islands on $\mathrm{Ag}(111)$, Phys. Rev. Lett. 76, 2113 (1996).

[44] M. Shen, C. J. Jenks, J. W. Evans, and P. A. Thiel, Rapid decay of vacancy islands at step edges on $\operatorname{Ag}(111)$ : Step orientation dependence, J. Phys.: Condens. Matter 22, 215002 (2010).

[45] P. B. Barna, G. Barcza, L. Tóth, G. Vincze, A. Bergauer, and H. Bangert, Structural evolution in codeposited Al-Sn thin films, Surf. Coat. Technol. 57, 7 (1993).

[46] P. B. Barna and M. Adamik, Growth Mechanisms of Polycrystalline Thin Films, In Science and Technology of Thin Films, edited by F. C. Matacotta (World Scientific Publishing, Singapore, 1995), pp. 1-28.

[47] P. B. Barna and M. Adamik, Fundamental structure forming phenomena of polycrystalline films and the structure zone models, Thin Solid Films 317, 27 (1998).

[48] R. Zhang and H. Huang, Another kinetic mechanism of stabilizing multiple-layer surface steps, Appl. Phys. Lett. 98, 221903 (2011).

[49] M. G. Lagally and Z. Zhang, Materials science: Thin-film cliffhanger, Nature (London) 417, 907 (2002).

[50] Y. Han, G.-H. Lu, B.-J. Lee, and F. Liu, Flat-surface, step-edge, facet-facet, and facet-step diffusion barrier in growth of a $\mathrm{Pb}$ mesa, Surf. Sci. 602, 2284 (2008).

[51] N. Combe, P. Jensen, and A. Pimpinelli, Changing Shapes in the Nanoworld, Phys. Rev. Lett. 85, 110 (2000).

[52] G. S. Rohrer, C. L. Rohrer, and W. W. Mullins, Nucleation energy barrier for volume-conserving shape changes of crystals with nonequilibrium morphologies, J. Am. Ceram. Soc. 84, 2099 (2001).

[53] J. Vrijmoeth, H. A. van der Vegt, J. A. Meyer, E. Vlieg, and R. J. Behm, Surfactant-Induced Layer-By-Layer Growth of Ag on $\mathrm{Ag}(111)$ : Origins and Side Effects, Phys. Rev. Lett. 72, 3843 (1994).

[54] E. Cox, M. Li, P.-W. Chung, C. Ghosh, T. S. Rahman, C. J. Jenks, J. W. Evans, and P. A. Thiel, Temperature dependence of island growth shapes during submonolayer deposition of $\mathrm{Ag}$ on Ag(111), Phys. Rev. B 71, 115414 (2005).

[55] M. Li, P.-W. Chung, E. Cox, C. J. Jenks, P. A. Thiel, and J. W. Evans, Exploration of complex multilayer film growth morphologies: STM analysis and predictive atomistic modeling for Ag on Ag(111), Phys. Rev. B 77, 033402 (2008).

[56] C. Busse, C. Polop, M. Müller, K. Albe, U. Linke, and T. Michely, Stacking-Fault Nucleation on Ir(111), Phys. Rev. Lett. 91, 056103 (2003)

[57] See Supplemental Material at http://link.aps.org/supplemental/ 10.1103/PhysRevMaterials.2.063401 for details on the bondcounting scheme, the adsorption-site energies, and comparison of the equilibrium island shapes with the Winterbottom construction.

[58] M. Müller, K. Albe, C. Busse, A. Thoma, and T. Michely, Island shapes, island densities, and stacking-fault formation on $\operatorname{Ir}(111)$ 
Kinetic Monte Carlo simulations and experiments, Phys. Rev. B 71, 075407 (2005).

[59] P. Zoontjens, T. P. Schulze, and S. C. Hendy, Hybrid method for modeling epitaxial growth: Kinetic Monte Carlo plus molecular dynamics, Phys. Rev. B 76, 245418 (2007).

[60] T. Michely and J. Krug, Islands, Mounds, and Atoms: Patterns and Processes in Crystal Growth Far from Equilibrium (Springer, Berlin, 2003), pp. 12-66.

[61] E. P. Münger, V. Chirita, J. E. Greene, and J.-E. Sundgren, Adatom-induced diffusion of two-dimensional close-packed $\mathrm{Pt}_{7}$ clusters on Pt(111), Surf. Sci. 355, L325 (1996).

[62] E. P. Münger, V. Chirita, L. Hultman, and J. E. Greene, Adatom/vacancy interactions and interlayer mass transport in small two-dimensional Pt clusters on Pt(111), Surf. Sci. 539, L567 (2003).

[63] M. Schmid, E. Lundgren, G. Leonardelli, A. Hammerschmid, B. Stanka, and P. Varga, Exchange processes interlayer diffusion kinks, corners and the growth mode, Appl. Phys. A 72, 405 (2001).

[64] J. Yu and J. G. Amar, Short-range attraction, surface currents, and mound formation in metal (111) epitaxial growth, Phys. Rev. B 69, 045426 (2004).

[65] W. K. Rilling, C. M. Gilmore, T. D. Andreadis, and J. A. Sprague, An embedded-atom-method study of diffusion of an Ag adatom on (111) Ag, Can. J. Phys. 68, 1035 (1990).

[66] G. Boisvert, L. J. Lewis, and A. Yelon, Many-Body Nature of the Meyer-Neldel Compensation Law for Diffusion, Phys. Rev. Lett. 75, 469 (1995).

[67] C. Ratsch, A. P. Seitsonen, and M. Scheffler, Strain dependence of surface diffusion: $\mathrm{Ag}$ on $\mathrm{Ag}(111)$ and $\mathrm{Pt}(111)$, Phys. Rev. B 55, 6750 (1997).

[68] C. Ratsch and M. Scheffler, Density-functional theory calculations of hopping rates of surface diffusion, Phys. Rev. B 58, 13163 (1998).

[69] H. Brune, K. Bromann, H. Röder, K. Kern, J. Jacobsen, P. Stoltze, K. Jacobsen, and J. Nørskov, Effect of strain on surface diffusion and nucleation, Phys. Rev. B 52, R14380 (1995).

[70] S. Y. Kim, I.-H. Lee, and S. Jun, Transition-pathway models of atomic diffusion on fcc metal surfaces. I. Flat surfaces, Phys. Rev. B 76, 245407 (2007).

[71] K. Morgenstern, G. Rosenfeld, E. Lægsgaard, F. Besenbacher, and G. Comsa, Measurement of Energies Controlling Ripening and Annealing on Metal Surfaces, Phys. Rev. Lett. 80, 556 (1998).

[72] M. Henzler, T. Schmidt, and E. Z. Luo, Modes in homoepitaxial growth on $\mathrm{Cu}(111), \operatorname{Ag}(111)$, and $\mathrm{Pt}(111)$, in The Structure of Surfaces IV, edited by X. Xie, S. Y. Tong, and M. A. V. Hove (World Scientific, Singapore, 1994), pp. 619-264.

[73] E. Z. Luo, J. Wollschläger, F. Wegner, and M. Henzler, SPALEED studies of growth of Ag on Ag (111) at low temperatures, Appl. Phys. A 60, 19 (1995).
[74] G. Rosenfeld, N. N. Lipkin, W. Wulfhekel, J. Kliewer, K. Morgenstern, B. Poelsema, and G. Comsa, New concepts for controlled homoepitaxy, Appl. Phys. A 61, 455 (1995).

[75] P. A. Thiel and J. W. Ewans, Nucleation, growth, and relaxation of thin films: Metal(100) homoepitaxial systems, J. Phys. Chem. B 104, 1663 (2000).

[76] L. Bardotti, C. R. Stoldt, C. J. Jenks, M. C. Bartelt, J. W. Evans, and P. A. Thiel, High-resolution LEED profile analysis and diffusion barrier estimation for submonolayer homoepitaxy of Ag/Ag(100), Phys. Rev. B 57, 12544 (1998).

[77] M. F. Roşu, C. R. Laurens, A. Falepin, M. A. James, M. H Langelaar, F. Pleiter, O. C. Rogojanu, and L. Niesen, Direct Observation of the Self-Diffusion Mechanism on the $\operatorname{Ag}(100)$ Surface, Phys. Rev. Lett. 81, 4680 (1998).

[78] S. Frank, H. Wedler, R. J. Behm, J. Rottler, P. Maass, K. J. Caspersen, C. R. Stoldt, P. A. Thiel, and J. W. Evans, Approaching the low-temperature limit in nucleation and two-dimensional growth of fcc (100) metal films $\mathrm{Ag} / \mathrm{Ag}(100)$, Phys. Rev. B 66, 155435 (2002).

[79] P. L. Williams, Y. Mishin, and J. C. Hamilton, An embeddedatom potential for the $\mathrm{Cu}-\mathrm{Ag}$ system, Model. Simul. Mater. Sci. Eng. 14, 817 (2006).

[80] R. Lesar, Introduction to Computational Materials Science: Fundamentals to Applications (Cambridge University Press, Cambridge, 2013), pp. 183-196.

[81] A. Stukowski, Visualization and analysis of atomistic simulation data with OVITO-the open visualization tool, Model. Simul. Mater. Sci. Eng. 18, 015012 (2010).

[82] S. Ogura, K. Fukutani, M. Matsumoto, T. Okano, M. Okada, and T. Kawamura, Dendritic to non-dendritic transitions in $\mathrm{Au}$ islands investigated by scanning tunneling microscopy and Monte Carlo simulations, Phys. Rev. B. 73, 125442 (2006).

[83] B. Lü, Ph.D. thesis, Linköping University, 2018.

[84] M. Hupalo, S. Kremmer, V. Yeh, L. Berbil-Bautista, E. Abram, and M. C. Trignides, Uniform island height selection in the low temperature growth of $\mathrm{Pb} / \mathrm{Si}(111)-(7 \times 7)$, Surf. Sci. 493, 526 (2001)

[85] Z. Kuntova, Z. Chvoj, M. C. Trignides, and M. Yakes, Monte Carlo simulation of growth modes of $\mathrm{Pb}$ nanoislands on $\mathrm{Si}(111)$ surface, Eur. Phys. J. B 64, 61 (2008).

[86] Y. Han, F. Liu, S.-C. Li, J.-F. Jia, and Q.-K. Xue, Kinetics of mesa overlayer growth: Climbing of adatoms onto the mesa top, Appl. Phys. Lett. 92, 021909 (2008).

[87] L. Gavioli, K. R. Kimberlin, M. C. Trignides, J. F. Wendelken, and Z. Zhang, Novel Growth of Ag Islands on Si(111): Plateaus with a Singular Height, Phys. Rev. Lett. 82, 129 (1999).

[88] R. B. Lewis, P. Corfdir, H. Li, J. Herranz, C. Pfüller, O. Brandt, and L. Geehaar, Quantum Dot Self-Assembly Driven by a Surfactant-Induced Morphological Instability, Phys. Rev. Lett. 119, 086101 (2017).

[89] A. A. Tonkikh and P. Werner, Surfactant-mediated StranskiKrastanov islands, Phys. Status Solidi B 250, 1795 (2013). 